\title{
Efficacy of Iron Oxide Nanoparticles in Diagnosis of Schistosomiasis
}

\author{
Ahmed Eid ${ }^{1}$ MBBCh, Khairy Abdel-Hamid ${ }^{2}$ MD, Mohamed Abdel-Hafez ${ }^{1}$ MD,
} Anwar Hosainy $^{2}$ MD, Ibrahim R Aly ${ }^{3 *}$ MD

\author{
*Corresponding Author \\ Ibrahim R Aly \\ ibrahimshalash@yahoo.com
}

Received for publication December 25, 2019; Accepted February 19, 2020; Published online January 22, 2020

Copyright 2020 The Authors published by Al-Azhar University, Faculty of Medicine, Cairo, Egypt. All rights reserved. This an open-access article distributed under the legal terms, where it is permissible to download and share the work provided it is properly cited. The work cannot be changed in any way or used commercially.

doi: 10.21608/aimj.2020.21461.1031

${ }^{1}$ Department of Medical Parasitology, Faculty of Medicine (Assiut), Al-Azhar University, Egypt.

${ }^{2}$ Department of Medical Parasitology, Faculty of Medicine, Al-Azhar University, Egypt.

${ }^{3}$ Department of Parasitology, Theodore Bilharz Research Institute, Giza, Egypt.

\begin{abstract}
Background: One of the worldwide major public health problems is urinary schistosomiasis that is caused by Schistosoma heamatobium. There are several immunodiagnostic methods used for that diagnosis of such disease, but some are more sensitive and specific than others. The tegumental Schistosoma-specific protein detection in serum samples is found out to be more valuable in diagnosis .

Aim of the Work: To evaluate the efficacy of iron oxide nanoparticle for diagnosis of human schistosomiasis infections and to compare between ELISA-based iron oxide nanoparticle and traditional sandwich ELISA.

Material and Methods: The tegumental antigen was purified from whole worms by DEAE-Sephadex G-75 ion-exchange chromatography and then was injected into rabbits to produce specific polyclonal antibodies ( $\mathrm{p} \mathrm{Ab}$ ) which were then used as a primary capture in the indirect ELISA technique to reveal its reactivity using infected human sera. The anti- tegumental $\mathrm{p}$ Ab was then labeled with horse-radish peroxidase (HRP) and used as a secondary capture. Sandwich ELISA was done for serum samples of humans and hamsters infected with $S$. haematobium.

Results: The sensitivity of the traditional sandwich ELISA with antitegumental $\mathrm{p} \mathrm{Ab}$ was $85 \%$ and it increased by using the sandwich IMBELISA to be $95 \%$ in serum. The specificity of sandwich ELISA was $88.2 \%$ and it increased by using the sandwich IMB-ELISA to be $92.6 \%$. Conclusion: The data obtained concluded that the IMB-ELISA appears to be a sufficiently sensitive and feasible assay for the detection of schistosomal antigenemia and the evaluation of its potential use in human schistosomiasis is in progress.

Keywords: S. haematobium; diagnosis; ELISA; Immunomagnetic bead ELISA technique; Paramagnetic nanoparticles.
\end{abstract}

Disclosure: The authors have no financial interest to declare in relation to the content of this article. The Article Processing Charge was paid for by the authors.

Authorship: All authors have a substantial contribution to the article

\section{INTRODUCTION}

Schistosomiasis, which is also known as bilharziasis, is one of the most worldwide spread disease-causing the highest rates of morbidity and mortality after malaria. Schistosomiasis infects about 200 million people worldwide. The contamination takes place by getting in contact with fresh water contaminated with cercaria which penetrates the skin causing infection. ${ }^{1}$ There are 3 main types of Schistosoma that cause human infections: S. mansoni, S. haematobium, or S.japonicum. ${ }^{2}$ In Egypt, the urinary schistosomiasis is representing a serious health problem to deal with. Its complications add more burden on the national control programs and on the national economy. ${ }^{3}$ Due to controlling programs over the last decade, a decline in the prevalence of human schistosomiasis in Egypt has been reported, however the disease is still endemic in many foci. ${ }^{4}$ Schematically, there are three different known approaches for the diagnosis of parasitic infections, direct (detecting parasitic ova in stool or urine), indirect (relaying on biochemical assays) and immunological methods which are the most sensitive and specific methods in measure the 
immune response (antibodies) to certain parasitic antigens and/or detecting circulating parasitic antigens. ${ }^{6}$ Proteases and peptidases are proteolytic enzymes that operate in virtually every biological phenomenon. They function not only as individual enzymes but often in cascades or networks. They also provide essential functions in all life forms. ${ }^{7}$ Proteases operate at the host-parasite interface facilitating migration, digestion of host proteins and probably immune evasion. 8,9

This study aimed to determine the sensitivity and specificity of ELISA-based iron oxide nanoparticles, evaluate the efficacy of iron oxide nanoparticlesfor diagnosis of human schistosomiasis infections and to compare between ELISAbased iron oxide nanoparticle and traditional sandwich ELISA.

\section{MATERIALAND METHODS}

Animals: New Zealand white male rabbits were purchased from Agriculture Faculty, Cairo University (Giza, Egypt), weighing approximately $1.5 \mathrm{Kg}$ and 2 months old. They were examined and confirmed to be free from parasitic infections. The rabbits were housed in the animal house at Theodore Bilharz Research Institute (TBRI) (Giza, Egypt), under standard laboratory care at $21^{\circ} \mathrm{C}, 16 \%$ moisture, the animals were supplied with filtered water with salts $1 \mathrm{~cm} / 5$ liter and vitamins $1 \mathrm{~cm} / 10$ liter, also their diet hold a minimum of $15 \%$ protein, $3 \%$ fat and $22 \%$ fiber. Internationally valid guidelines were applied to animal experiments.

Parasites: Adult worms of Schistosoma haematobium used for the preparation of purified tegumental antigen were supplied from the Schistosome Biological Supply Program Unit at Theodore Bilharz Research Institute (SBSP, TBRI) Giza, Egypt. Adult worms were recovered from the portal mesenteric vasculatures of laboratory infected hamsters by perfusion with heparinized saline as previously described by Smithers and Terry. ${ }^{10}$

Study population: A total of 128 individuals were enrolled in the present study. Thirty individuals were free from any parasitic infections served as normal control. Sixty patients infected with $S$. haematobium and 38 patients infected with other helminthic parasites (It included 10 patients infected with $H$. nana, 8 with Fasciolagigantica, 8 with Ascarislumbricoides and 12 with Ancylostomaduodenale).

Preparation and purification of tegumental from $S$. haematobium.

\section{Homogenization:}

Freshadult

worms

ofSchistosoma

haematobiumweresuspended in $10 \mathrm{ml}$ of phosphate buffer saline (PBS) anddialyzed against lysis buffer [8 M Urea, 2MThiourea,4\%3,3,Cholamidopropyl,dimethylammonium, propanesulfonate (CHAPS),50 Mdithiothreitol (DTT),20 M MTris and complete Mini Protease Inhibitor Cocktail Tablets (Roche)].After dialysis, the adult worms were homogenizedunder continuous agitation for 2 hours at room temperature

witha glasshomogenizer,followed by 10 repeated passagesthrough a 30-gauge hypodermicneedle.Thenthe homogenate was centrifuged at $20,000 \times \mathrm{g}$ for $30 \mathrm{~min}$ at $25^{\circ} \mathrm{C}$ and the supernatant was collected and stored at $-70^{\circ} \mathrm{C}$.

Purification of schistosomal antigen was done by DEAE Sepharose CL-6B and the protein content was estimated by the Bio-Rad kit. Characterization of schistosomal antigen was done using Sodium dodecyl-sulfate polyacrylamide gel electrophoresis (SDS-PAGE) according to Harlow \& Lane $^{11}$ and Myers. ${ }^{12}$

Assessment of reactivity of the schistosomal antigen of S.haematobium by indirect ELISA: This method was performed, with some modifications from the original method of Engvall and Perlmann. ${ }^{13}$

Immunization of rabbit for production of polyclonal antibodies: Rabbit anti-serum was obtained by immunizing New Zealand white rabbit (approximately $1.5 \mathrm{Kg}$ weight) with $1 \mathrm{mg}$ of purified schistosomal antigen that was given to the rabbit in the entire course of immunization. The rabbit received priming dose intramuscular injection (i.m) at four sites (1mg purified schistosomal antigen mixed 1:1 incomplete Freund's adjuvant (CFA), (Sigma).

Three booster doses were given, each was $0.5 \mathrm{mg}$ antigen emulsified in equal vol. of incomplete Freund's adjuvant (IFA), (Sigma). The first boosting was two wk. after the priming dose. The following boosting doses were given at weekly intervals, according to Fagbemi. ${ }^{14}$

The rabbit was bled for collection of serum one week later after preliminary testing of titer by indirect ELIZA.

Purification of rabbit anti- purified schistosomal antigen serum: Rabbit IgG purification steps were based on two different methods: Ammonium sulfate precipitation method $^{15}$ and caprylic acid treatment. ${ }^{16}$

Protein content was measured after each purification step using the Bradford method. ${ }^{17}$ The efficiency of the purification steps was measured by $12 \%$ SDS-room temperature. The plates were washed 5 times with

washing buffer. Hundred $\mu \mathrm{l} /$ well of substrate solution [one tablet of OPD (Sigma) dissolved in $25 \mathrm{ml}$ of $0.05 \mathrm{M}$ phosphate citrate buffer, pH 5 with urea hydrogen peroxidase (Sigma)] was added to each well and the plates were incubated in the dark at room temperature for $30 \mathrm{~min}$. Fifty $\mu \mathrm{l} /$ well of $8 \mathrm{~N} \mathrm{H}_{2} \mathrm{SO}_{4}$ was added to stop the enzymesubstrate reaction. The absorbance was measured at $492 \mathrm{~nm}$ using ELISA reader (Bio-Rad microplate reader Richmond, $\mathrm{Ca}$ ).

Statistical analysis: Data are expressed as mean (M) \pm standard deviation (SD). Statistical analysis was performed with the aid of the SPSS computer program (version windows 16.0).

\section{RESULTS}

Characterization of $S$. haematobium membrane antigen by (SDS-PAGE)

The protein fractions resulted from the preparation methods were analyzed by $12.5 \%$ SDS-PAGE under reducing condition and showed 2 major at 65 and $95 \mathrm{KDa}$ and many minor bands representing $S$. haematobium membrane antigen (Fig. 1). 


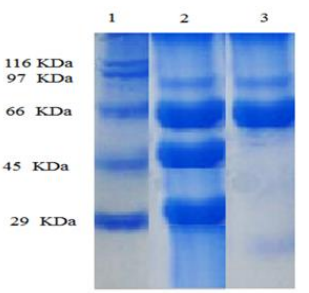

Fig. 1: SDS-PAGE of $S$. haematobium membrane antigen Lane 1: Molecular weight of the standard protein

Lane 2: Crude $S$. haematobium antigen

Lane 3: Purified $S$. haematobium membrane antigen

Assessment of reactivity and specificity of the prepared S. haematobium membrane antigen by indirect-ELISA

The antigenicity of the $S$. haematobium membrane antigen was tested by indirect ELISA technique. Serum samples from an infected human with $S$. haematobium gave a strong reaction against $S$. haematobium membrane antigen with mean OD reading equal to 0.929 and no cross-reactions were recorded with sera of patients infected with other parasites e.g., Fasciola, H. nana and Ascaris (Table 1).

\begin{tabular}{|l|c|}
\hline \multicolumn{1}{|c|}{ Serum Samples } & $\begin{array}{c}\text { OD readings at 492 } \\
\mathrm{nm}(\mathrm{M} \pm \mathrm{SD})\end{array}$ \\
\hline S. haematobium & $0.929 \pm 0.042$ \\
\hline Fasciola gigantica & $0.291 \pm 0.071$ \\
\hline H.nana & $0.18 \pm 0.011$ \\
\hline Ascaris lumbricoides & $0.24 \pm 0.056$ \\
\hline
\end{tabular}

$\mathrm{OD}=$ optical density, $\mathrm{SD}=$ standard deviation

Table 1: Reactivity of $S$. haematobium membrane antigen by indirect ELISA

pAb titers detection and testing for reactivity against $S$. haematobium membrane antigen by indirect ELISA

An increasing antibody level started $1 \mathrm{wk}$ after the first booster dose. Three days after the $2^{\text {nd }}$ booster dose immune sera gave a high titer against $S$. haematobium membrane antigen with OD of 2.97 at 1/250 dilution (Fig. 2).

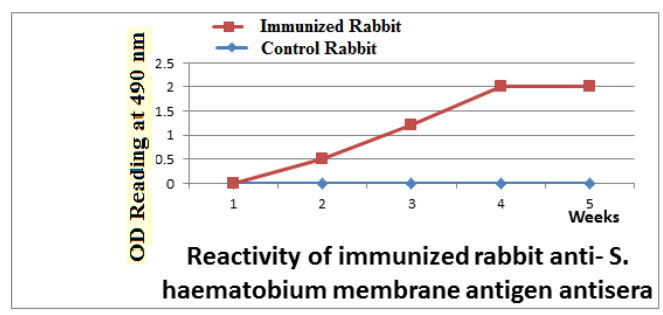

Fig. 2: Reactivity of immunized rabbit anti- S. haematobium membrane antigen antisera (diluted $1 / 250$ ) against $S$. haematobium membrane antigen by indirect ELISA

Protein content determination of the purified antiS.haematobiumIgG-pAb

The total protein content of the rabbit's crude serum- containing anti-S.haematobiumpAb was $7.2 \mathrm{mg} / \mathrm{ml}$. Using the $50 \%$ ammonium sulfate precipitation method, the protein content was $5.1 \mathrm{mg} / \mathrm{ml}$, while following a $7 \%$ caprylic acid precipitation method, the content dropped to $3.5 \mathrm{mg} / \mathrm{ml}$. Finally, the protein content of the highly purified antiS.haematobium IgG-pAb subjected to ion-exchange chromatography method (DEAE Sephadex A-50 ion exchange column chromatography) was $2.1 \mathrm{mg} / \mathrm{ml}$.

Figure (3) shows the $\mathrm{OD}_{492}$ profile of the $\mathrm{IgG}$ fractions obtained following purification by DEAE Sephadex A-50 ion-exchange column chromatography. The eluted IgG is represented by a single peak with a maximum OD value 0.48 at fraction number 17.

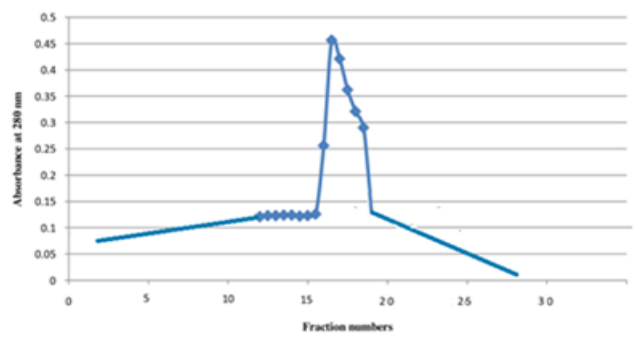

Fig. 3: $\mathrm{OD}_{280}$ profile of fraction obtained following rabbit's antiS.haematobium IgG-pAb purification by DEAE ion-exchange chromatography

\section{Characterization of anti-S.haematobium IgG-pAb by} SDS-PAGE

The purity of IgG-pAb after each step of purification was assayed by $12 \%$ SDS-PAGE under reducing conditions. Analysis of $50 \%$ ammonium sulfate-precipitated proteins by $12 \%$ SDS-PAGE under reducing conditions showed that precipitated proteins appeared as several bands.

The purified IgGpAb after ion exchange chromatography was represented by only 2 bands, Land $\mathrm{H}$-chain bands at 31 and $53 \mathrm{kDa}$, respectively. The pAb appears free from other proteins (Fig. 4).

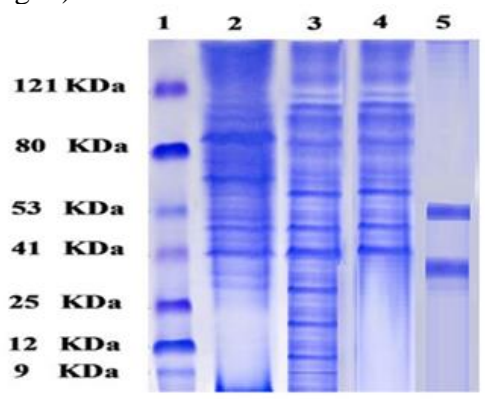

Fig. (4): $12.5 \%$ SDS-PAGE of anti-S. haematobiumIgG-pAb before and after purification

Lane 1: Molecular weight of the standard protein

Lane 2: Crude anti- S. haematobium membrane antigen IgG-pAb (before purification)

Lane 3: Precipitated proteins after 50\% ammonium sulfate treatment

Lane 4: Purified IgG-pAb after 7\% caprylic acid treatment Lane 5: Purified IgG-pAb after ion-exchange chromatography.

Assessment of specificity of the purified pAb by indirect 


\section{ELISA}

The produced anti- $S$. haematobium membrane antigenIgGpAb diluted $1 / 250$ in PBS/T buffer gave strong reactivity to $S$. haematobium membrane antigen. The OD means reading at $492 \mathrm{~nm}$ for $S$. haematobium membrane antigen was 2.84 compared to 0.24, 0.19, 0.31 and 0.41 for Fasciolia, H.nana, Ancylostoma and Ascaris infected sera, respectively (Table 2).

\begin{tabular}{|l|c|}
\hline Parasitic antigen & $\begin{array}{c}\text { OD readings at } \\
\mathbf{4 9 2} \mathbf{~ n m}(\mathbf{m} \pm \mathbf{S D})\end{array}$ \\
\hline S. haematobium & $2.84 \pm 0.21$ \\
F. gigantica & $0.24 \pm 0.13$ \\
\hline H.nana & $0.19 \pm 0.11$ \\
\hline A. duodenale & $0.31 \pm 0.14$ \\
\hline A.lumbricoides & $0.41 \pm 0.10$ \\
\hline E. vermicularis & $0.18 \pm 0.13$ \\
\hline
\end{tabular}

Table 2: Specificity of rabbit anti- S. haematobium membrane antigen IgG-pAb against different parasitic antigens by indirect ELISA

\section{Detection of $S$. haematobium membrane antigen in serum samples by sandwich ELISA}

The cut off value was 0.342 . When detecting $S$. haematobium membrane antigen in serum, the results were positive in 01 cases $(80 \%)$ of group A, while 9 cases were negative (10\%). In group B (patients with other parasitic infections) $\wedge$ cases were detected as positive $(r$ with Fasciola, ${ }^{\top}$ withAncylostoma infection, and $r$ with $H$. nana infection), while the other 3 - cases were negative. All healthy control patients were negative. The sensitivity of $S$. haematobium membrane antigen detection in serum was found to be $80 \%$. However, the specificity was found to be $\wedge \wedge, \uparrow \%$ in the healthy control group and in the patients with other parasitic infections. There is a highly statistically significant difference between the positivity in $S$. haematobium infected group and the other two tested groups $(\mathrm{P}<0.001)($ Table 3$)$.

\begin{tabular}{|c|c|c|c|c|}
\hline \multirow{2}{*}{ GROUPS } & \multicolumn{2}{|c|}{ Positive cases } & \multicolumn{2}{|c|}{ Negative cases } \\
\hline & (n.) & $\mathrm{OD}(\mathrm{X} \pm \mathrm{SD})$ & (n.) & $\mathrm{OD}(\mathbf{X} \pm \mathrm{SD})$ \\
\hline $\begin{array}{c}\text { Healthy } \\
\text { control } \\
(\mathrm{n}=30)\end{array}$ & - & - & 30 & $0.321 \pm 0.02$ \\
\hline $\begin{array}{c}\text { S.haemtobium } \\
\quad(\mathrm{n}=60)\end{array}$ & 51 & $1.15 \pm 0.12$ & 9 & $0.303 \pm 0.05$ \\
\hline $\begin{array}{c}\text { Fasciola } \\
(\mathbf{n}=\mathbf{8})\end{array}$ & 3 & $0.424 \pm 0.03$ & 5 & $0.228 \pm 0.06$ \\
\hline $\begin{array}{c}\text { H.nana } \\
(\mathrm{n}=8)\end{array}$ & 3 & $0.543 \pm 0.06$ & 5 & $0.228 \pm 0.04$ \\
\hline $\begin{array}{r}\text { Ascaris } \\
(\mathrm{n}=10\end{array}$ & 0 & - & 10 & $0.274 \pm 0.08$ \\
\hline $\begin{array}{c}\text { Ancylostoma } \\
(\mathrm{n}=12)\end{array}$ & 2 & $0.701 \pm 0.03$ & 10 & $0.262 \pm 0.04$ \\
\hline
\end{tabular}

Table 3: Results of S. haematobium membrane antigen detection in serum samples by sandwich ELISA

\section{Detection of $S$. haematobium} membrane antigen in serum samples conjugated with IO by sandwich IMB -ELISA

The calculated cut off OD value was 0.282 . The presence of $S$. haematobium membrane antigen in serum samples of the different studied groups was evaluated by the nanomagnetic beads method. The mean OD value of the $S$. haematobium infected group $(1.97 \pm 0.22)$ was significantly higher than that of other parasites group (group B).

Out of 60 schistosomiasis cases, 57 cases gave positive results, while 3 cases gave negative results, giving a sensitivity of $95 \%$. All the 30 healthy controls (group C) were negative being below the cut off value for $S$. haematobium membrane antigen positivity giving a $100 \%$ specificity. In group B (patients with other parasitic infections), only 5 cases were detected as positive (2 with Fasciola, 1 with Ascarislumbricoides, 1 case with $H$. nana and 1 case withAncylostomaduodenale infection), while the other 33 cases were negative giving specificity of the procedure of $92.6 \%$ to group B. The P-value was < 0.001 which means that there is a statistical significance in positivity between $S$. haematobiuminfectedgroup and other tested groups (Table 4).

\begin{tabular}{|c|c|c|c|c|}
\hline \multirow{2}{*}{ GROUPS } & \multicolumn{2}{|c|}{ Positive cases } & \multicolumn{2}{|c|}{ Negative cases } \\
\hline & (n.) & OD $(\mathrm{X} \quad \pm \mathrm{SD})$ & (n.) & $\mathbf{O D}(\mathbf{X} \pm \mathrm{SD})$ \\
\hline $\begin{array}{c}\text { Healthy } \\
\text { control } \\
(\mathrm{n}=30)\end{array}$ & - & - & 30 & $0.321 \pm 0.02$ \\
\hline $\begin{array}{c}\text { S.haemtobium } \\
(\mathrm{n}=60)\end{array}$ & 57 & $1.15 \pm 0.12$ & 3 & $0.303 \pm 0.05$ \\
\hline $\begin{array}{c}\text { Fasciola } \\
(\mathrm{n}=8)\end{array}$ & 2 & $0.424 \pm 0.03$ & 6 & $0.228 \pm 0.06$ \\
\hline $\begin{array}{c}\begin{array}{c}\text { H.nana } \\
(\mathrm{n}=8)\end{array} \\
\text { (n) }\end{array}$ & 1 & $0.543 \pm 0.06$ & 7 & $0.228 \pm 0.04$ \\
\hline $\begin{array}{c}\text { Ascaris } \\
(\mathrm{n}=10)\end{array}$ & 1 & - & 9 & $0.274 \pm 0.08$ \\
\hline $\begin{array}{c}\text { Ancylostoma } \\
(\mathrm{n}=12)\end{array}$ & 1 & $0.701 \pm 0.03$ & 11 & $0.262 \pm 0.04$ \\
\hline
\end{tabular}

Table 4: Results of S. haematobium membrane antigen detection in serum samples conjugated with IO by sandwich IMB-ELISA

Table (4) shows the incidence of positivity for antigen detection in human sera by the two diagnostic techniques used in the study. The two techniques illustrated a high percentage of positivity reaching $100 \%$ in serum of the high infection subgroup. In the moderate infection subgroup, the incidence of positivity for antigen in serum was $80 \%, 92 \%$, $84 \%$ and $96 \%$ by sandwich ELISA, sandwich IMB-ELISA, respectively. Whereas, in the light infection subgroup, the incidence of positivity for antigen in serum was $80 \%, 92 \%$, $84 \%$ and $96 \%$ by sandwich ELISA, sandwich IMB-ELISA, respectively.

The other parasites-infected groups mainly with Fasciola gigantica showed some degree of cross-reactivity with antigens in human sera.

\section{DISCUSSION}

Schistosomiasis is one of the main occupational diseases, acquired by man through activities associated with freshwaters such as farming, washing, bathing, and recreation. It has been recognized as a disease of significant socio-economic and public health importance second to malaria ${ }^{20}$. Urinary schistosomiasis remains a major health burden in endemic areas of Africa and the Middle East, 
affecting more than 110 million people in rural, agricultural and peri-urban areas ${ }^{\mathbf{2 1}}$. Estimates show that at least 220.8 million people required preventive treatment in 2017, out of which more than 102.3 million people were reported to have been treated. Preventive treatment, which should be repeated over a number of years, will reduce and prevent morbidity. Schistosomiasis transmission has been reported from 78 countries. However, preventive chemotherapy for schistosomiasis, where people and communities are targeted for large-scale treatment, is only required in 52 endemic countries with moderate-to-high transmission. ${ }^{20}$

In Egypt, schistosomiasis is still representing a serious health problem to deal with. Its complications add more burden to the national control programs and on the national economy. Due to control programs over the last decade, a decline in the prevalence of human schistosomiasis in Egypt has been reported, however, the disease is still endemic in many foci.

${ }^{3,4}$ Schistosomiasis haematobium was highly prevalent $(60 \%)$ both in the Nile Delta and Nile Valley South of Cairo in districts of perennial irrigation while it was low $(6 \%)$ in districts of basin irrigation. Schistosoma mansoni infected $60 \%$ of the population in the Northern and Eastern parts of the Nile Delta and only $6 \%$ in the Southern part. ${ }^{21,22}$

The present work aimed to evaluate the efficacy of iron oxide nanoparticles for diagnosis of human Schistosomiasis infections, determine the sensitivity and specificity of ELISAbased iron oxide nanoparticles and compare between ELISAbased iron oxide nanoparticles and traditional sandwich ELISA.

The two Schistosomiasis antigens were used in the production of anti-Schistosomiasis IgG antibodies. Their antigenicity was tested by indirect ELISA technique and purified Schistosomiasis antigens gave positive reaction against Schistosomiasis infected sera and no cross-reactions against other parasites sera. The stronger reactivity was in favor of purified antigen.

The recorded sensitivity of sandwich ELISA using anticrude Schistosomiasis IgG alone or conjugated with IOPAbs was $85 \%$ and $95 \%$, respectively and their specificities were $88.3 \%$ and $93.3 \%$, respectively. As noted, higher diagnostic indices (sensitivity and specificity) were recorded in IO conjugated IgGs than traditional IgGs ELISA models. In this study, the use of IO conjugated with IgG led to increasing the number of their binding sites and adsorptive capacity. This was attributed to their small size. Many authors used novel nano-diagnostic assays in the diagnosis of many parasitic infections, e.g., Schistosoma japonicum ${ }^{23}$, Schistosoma haematobium. ${ }^{24}$

Lei et al. ${ }^{23}$ developed ELISA assay using IgYpAb-coated with a magnetic bead as a capture $\mathrm{Ab}$ and $\mathrm{IgGm} \mathrm{Ab}$ as antigen-detection $\mathrm{Ab}$ against S.haematobium. The recorded sensitivity was $100 \%$ and $91.5 \%$ (in acute and chronic infection, respectively) and specificity $96.7 \%-100 \%$ with clonorchiasis and paragonimiasis positive cases.

Ibrahim et al. ${ }^{24}$ also developed a novel IMB-ELISA based IgG for the detection of excretory/secretory antigens in rabbit sera infected with $S$. haematobium. The reported sensitivity and specificity of the assay were $95 \%$ and $93.7 \%$. They used magnetic microbeads based-sandwich ELISA for the detection of $S$. haematobium circulating microsomal fraction antigen. The reported sensitivity and specificity of the assay were $96.5 \%$ and $96.3 \%$ compared to $88.2 \%$ and $87.3 \%$ by traditional sandwich ELISA.

It was noted that the application of the nanomagnetic beads significantly increased the sensitivity, specificity, and incidence of positivity of the technique towards higher detection of the antigen in moderate and light infected patients. The sensitivity of the traditional sandwich ELISA was $85 \%$ in serum and it increased by using the sandwich IMB-ELISA to be $95 \%$ in serum. The specificity of sandwich ELISA was $88.3 \%$ in serum and it increased by using the sandwich IMB-ELISA to be $93.3 \%$ in serum.

\section{CONCLUSION}

The data obtained concluded that the IMB-ELISA appears to be a sufficiently sensitive and feasible assay for the detection of schistosomal antigenemia and the evaluation of its potential use in human schistosomiasis needs more research.

\section{REFERENCES}

1- Liu Y, Zhou Y, Yang Y et al. Epidemiological features and effectiveness of schistosomiasis control program in the mountainous and hilly region of The People's Republic of China. Adv. Parasitol. 2016; 92:73-95.

2- CDC. Parasites-Schistosomiasis: Epidemiology and Risk Factors.

2012.http://www.cdc.gov/parasites/schistosomiasis/epi. html.

3- EL-Khoby T, Galal N, Barakat $\mathrm{R}$ et al. The epidemiology of schistosomiasis in Egypt:Summary findings in nine Governorates. Am. J. Trop. Med. Hyg. 2000; 62:88-99.

4- Bergquist N, Johansen M and Utzinger, J. Diagnostic dilemmas in helminthology: what tools to use and when? Trends Parasitol. 2009; 4:151-156.

5- Kandeel A, Genedy M, El-Refai S et al. The prevalence of HCV infection in Egypt 2015: Implications for future policy on prevention and treatment. Liver Int. 2017;37:45-53.

6- Bruschi $\mathrm{F}$ andCastagna B.The serodiagnosis of parasitic infections. Parassitologia;2004. 46,141-144.

7- Turk V, Stoka V, Vasiljeva O, et al.Cysteine cathepsins: From structure, function, and regulation to new frontiers: Proteins and BiochimicaBiophysicaActa.2012; 1824: 68-88.

8- Mckinney $\mathrm{M}$ and Parkinson A. A simple, nonchromatographic procedure to purify immunoglobulins from ascites fluid. J. Immunol. Meth., 1987;96:271-278.

9- Dalton J, Caffrey C, Sajid M, et al. Proteases in trematode biology. In: Parasitic Flatworms: Molecular Biology, Biochemistry, Immunology, and Physiology. 2006; Maule AG, Marks NJ, editors. Wallingford: CAB.

10-Smithers S and Terry R. The infection of laboratory hosts with cercariae of Schistosomamansoni and the recovery of the adult worms. Parasitology;1965; 55:695-700. 
11- HarlowE and Lane D. Antibodies, a laboratory manual. Cold Spring Harbor Laboratory, Cold Spring Harbor, New York.1988.

12- Myers R.Immunology, a laboratory manual. ( $2^{\text {nd }}$ ed); Chapter 2 Animal and Human Immunoglobulins Academic Press, New York.1995.

13- Engvall E and Perlman P.Enzyme-linked immunosorbent assay (EL1SA). Quantitative assay of characterization G. J. Immunochem., 1971;8:871-874.

14- Fagbemi B. Development and characterization of a monoclonal antibody reactive with $128 \mathrm{kDa}$ protease of F. gigantica. Vet. Parasitol.,1995;57: 351-354.

15- Nowotny A. Basic Exercises in immunochemistry. Springer Verlag, Berlin Heidelberg. New York. 1979;Pp: 7-20.

16- McKerrow J, CaffreyC, Kelly B et al. Proteases in parasitic diseases. Ann. Rev. Pathol,2006;1:497-536

17- Bradford M. A rapid and sensitive method for the quantitation of microgram quantities of protein utilizing the principle of protein-dye binding. Anal.Biochem.1976;72:245-254.
18- Laemmli V.Cleavage of structural proteins during the assembly of the head of the bacteriophage T4. Nature., 1970;277: 630-634.

19- Tijssen $P$ andKurstak P.Highly efficient and simple methods for the preparation of peroxidase and active peroxidase-antibody conjugate for enzyme immunoassays. Anal. Biochem., 1984;136: 451-457.

20- WHO. Egypt leverages domestic funding to eliminate schistosomiasis, 2019.

21- Chitsulo L, Loverde P andEngels D. Schistosomiasis. Nat. Rev.Microbiol., 2004; 2: 12-23.

22-Rashida $M$ and Barakat $M$. Epidemiology of Schistosomiasis in Egypt: Travel through Time: Review. Journal of Advanced Research,2013. 4 (5):425-432 .

23- Lei J, Liu W, Sun C, et al. Detection of circulating antigen in serum of mice infected with Schistosoma japonicum by immunomagnetic bead ELISA based on IgY.Acta Trop.,2009 111(1):39-43.

24- Aly I, Zalat R, El AswadB, et al.Novel Nanomagnetic Beads Based - Latex Agglutination Assay for Rapid Diagnosis of Human Schistosomiasis Haematobium. International Journal of Medical and Health Sciences;2013;7(12):977-81 